urgent radiology referrals rather than all being allocated to TWR appointments amenable to direct GP bookings. More work is needed to develop this referral pathway further.

\section{P231 "SAFETY-NET" FOR ABNORMAL CHEST RADIOGRAPHS WITH A LOW INDEX OF SUSPICION FOR MALIGNANCY}

doi:10.1136/thoraxjnl-2011-201054c.231

C Wall, P Arora, P M Turkington, R Chisholm, P M Turkington. Salford Royal NHS Foundation Trust, Manchester, UK

Introduction and Objectives Guidelines dictate that where a chest Xray is incidentally suggestive of lung cancer, a copy of the radiologists report is sent to a designated member of the lung cancer multidisciplinary team. A similar system was implemented for abnormal chest radiographs demonstrating significant non-malignant changes or abnormalities with a low index of suspicion of malignancy. This was used to assess if abnormal chest radiographs are adequately followed-up by the requesting clinicians.

Methods Reporting radiologists coded radiographs that warranted further action such as repeat imaging within a specified time frame. These were relayed to a designated coordinator. If action did not occur as suggested on the report, the requesting clinician was prompted on a weekly basis until the issue was resolved or else referred to a member of the chest team for further evaluation. At 6 months the cases were reviewed and information extracted including indication for further action, whether action occurred without coordinator intervention and outcome.

Results All coded films over a 6 -month period $(n=331)$ were analysed. The most frequent indications for further action included the assessment of pneumonia resolution $(n=167)$, further review of possible hilar pathology $(n=25)$, small nodules $(n=22)$ and other unexplained abnormalities. 143 cases (42.6\%) required action by the coordinator. In total, $4 \%(n=13)$ of cases yielded significant diagnoses including eight malignancies, pulmonary embolism, cobalt induced fibrosis and asbestosis, of these one case required action by the coordinator.

Conclusions This study suggests that a large proportion of abnormal chest radiographs are not followed-up as suggested by the reporting radiologist. Significant diagnoses may therefore be delayed unnecessarily. There is little reason to assume that similar practice is uncommon throughout the country. While the number of significant diagnoses detected by the "safety-net" system was perhaps lower than expected, some may consider a delay in $7.7 \%(1 / 13)$ unacceptably high. Further exploration of this area is needed to decide whether this is an effective use of resources.

\section{P232 SHORT-TERM OUTCOMES IN HEART FAILURE PATIENTS WITH CHRONIC OBSTRUCTIVE PULMONARY DISEASE IN THE COMMUNITY}

doi:10.1136/thoraxjnl-2011-201054c.232

${ }^{1} \mathrm{~S}$ Ghosh, ${ }^{2} \mathrm{~N} 0$ Kelly, ${ }^{2} \mathrm{~J}$ Smith, ${ }^{2} \mathrm{~J}$ Dexter, ${ }^{2} \mathrm{C}$ Carroll-Hawkins, ${ }^{1} \mathrm{~W}$ Robertson. ${ }^{1}$ University of Leicester, Leicester, UK; ${ }^{2}$ Leicestershire Partnership NHS Trust, Leicestershire, UK

Aims Heart failure (HF) and chronic obstructive pulmonary disease (COPD) are common co-morbidities. The combination presents diagnostic challenges and has been linked with worse prognosis in patients admitted to hospital. There is hardly any prognostic data in patients with both co-morbidities in the community.

Methods and Results We evaluated 783 patients (27.2\%) with left ventricular systolic dysfunction under the care of a regional nurse-led community HF team between June 2007 and June 2010. 101 patients $(12.9 \%)$ also had a diagnosis of COPD. $94 \%$ of patients were on loop diuretics; $83 \%$ on ACE inhibitors, $74 \%$ on $\beta$-blockers; $10.6 \%$ were on bronchodilators and $42 \%$ on aldosterone antagonists. Mean age of the patients was $77.9 \pm 5.7$ years; $43 \%$ were females and mean NYHA class was $2.3 \pm 0.6$. Mean follow-up was $28.2 \pm 2.9$ months. $\beta$-Blocker utilisation was markedly lower in patients receiving bronchodilators compared to those without (overall $21.7 \%$ vs $81 \%$; $p<0.001$ ). 24 month survival (Abstract P232 figure 1) was $93 \%$ in patients with HF alone and $89 \%$ in those with both co-morbidities $(p=N S)$. The presence of COPD was associated with increased HF hospitalisations [HR 1.56 (1.4 to 2.1); $\mathrm{p}<0.001$ ] and major adverse cardiovascular events [HR 1.23 (1.03 to 1.75); $\mathrm{p}<0.001$ ].

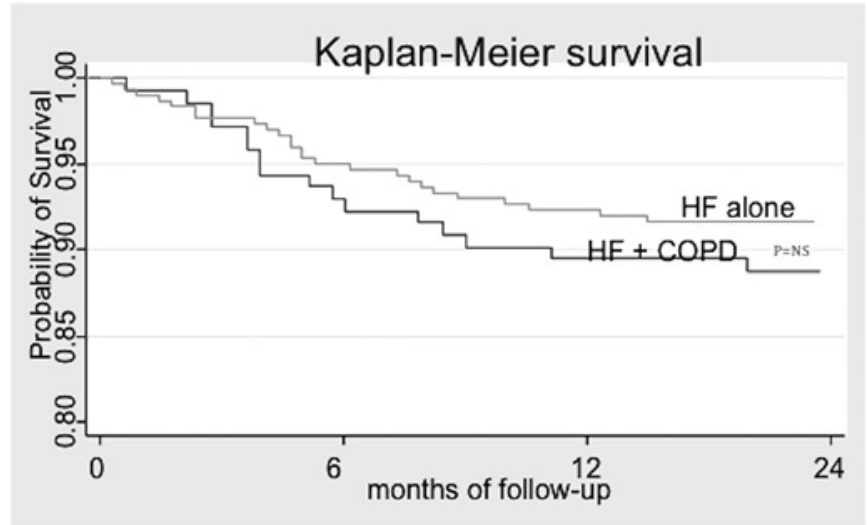

Abstract P232 Figure 1 Kaplan-Meier survival curve in patients with heart failure, comparing those with and without chronic obstructive pulmonary disease for June 2007 to June 2010.

Conclusions COPD is a common co-morbidity in ambulatory HF patients in the community and is a powerful predictor of worsening HF. It does not however appear to affect short-term mortality in ambulatory HF patients.

\section{P233 JUDICIOUS USE OF OXIMETRY CAN HELP DELIVER COST EFFECTIVE SLEEP SERVICE}

doi:10.1136/thoraxjn|-2011-201054c.233

C L Collins, B Balakrishnan, J Madieros. Queen's Medical Centre, Nottingham University Hospitals, Nottingham, UK

Background Referrals to sleep service are increasing rapidly and make up to $30 \%$ of referrals to the Queen's Medical Centre campus of Nottingham Respiratory Department. Limited channel polysomnography (PSG) at home (PSH-H) or video (PSG V) are time consuming and costly. In a retrospective study we assessed the role of oximetry in delivering cost effective sleep service.

Methods We reviewed new referrals to sleep clinic during February and March 2011. We aimed to quantify referrals, assess what investigations were done, the results of these and subsequent management. Cost analysis was then performed to assess impact of the tests ordered on service delivery. Costs of various tests at our centre are: oximetry $£ 28$, PSG- $\mathrm{H}-£ 200$ and PSG-V—£350.

Results In February and March this year 79 new referrals were identified for snoring or sleep disturbance. 53 patients (67\%) had oximetry as first test and 26 (33\%) had PSG-H or PSG limited or video PSG. The two groups had similar baseline characteristics with BMI of and Epworth Sleepiness Scale (ESS) score of 11. Of those who initially had oximetry 15 (29\%) went on to have PSG. Thus $52 \%$ of all referrals had PSG whereas $48 \%$ were managed with oximetry only. There was statistically significant correlation between $4 \%$ oxygen desaturation index per hour (4\% ODI) and apnoea-hypopnoea index (AHI) obtained from PSG $(\mathrm{R}=0.96 ; \mathrm{p}=0.05)$. The type of diagnostic test used had no impact on CPAP use. We see 600 new referrals per year. Had all patients been subjected to PSG it would cost the service 